\title{
Needlelike motion of prolate ellipsoids in the sea of spheres
}

\author{
R. Vasanthi \\ Solid State and Structural Chemistry Unit, Indian Institute of Science, Bangalore, India 560012 \\ S. Ravichandran \\ Department of Chemistry and Biochemistry, Duquesne University, Pittsburgh, Pennsylvania 15282 \\ Biman Bagchia) \\ Solid State and Structural Chemistry Unit, Indian Institute of Science, Bangalore, India 560012
}

\begin{abstract}
Molecular dynamics simulations of translational motion of isolated prolate ellipsoids in the sea of spheres have been carried out for several different values of the aspect ratio $(\kappa)$, obtained by changing either the length or the diameter of the ellipsoids, at several different solvent densities. The interaction among the spheres is given by the Lennard-Jones pair potential while that between spheres and ellipsoids is given by a modified Gay-Berne potential. Both the mean-square displacements of the center of mass of the ellipsoids and their orientational time correlation function have been calculated. It is found that at short to intermediate times, the motion of ellipsoids is anisotropic and primarily needlelike- the molecules prefer to move parallel to their long axis. The ratio of these two diffusion constants $\left(D_{\|}\right.$and $\left.D_{\perp}\right)$ approaches $\kappa$, suggesting a decoupling of $D_{\|}$ from the length of the ellipsoid. The diffusion becomes isotropic in the long time with the total diffusion coefficient given by $D_{\|}+2 D_{\perp}$. The crossover from the anisotropic to the isotropic diffusion is surprisingly sharp and clear in most cases.
\end{abstract}

\section{INTRODUCTION}

In many systems of interest in physics, chemistry, and biology, molecules are highly anisotropic in shape. ${ }^{1}$ Extreme examples are provided by rod-like molecules, such as tobacco mosaic virus. ${ }^{2,3}$ The translational motion of these molecules can be quite different from those of spherical or nearly spherical molecules. Surprisingly, not much is known about the motion of these highly anisotropic molecules in liquids -neither experimental nor many simulation studies have been carried out to investigate the translational motion of these molecules. The only information seems to come from the solution of Navier-Stokes hydrodynamic equations which provide expressions for the friction on ellipsoidal molecules. The hydrodynamic solutions provide two dramatically opposite scenarios depending on whether the stick or the slip boundary condition is applied. The Stokes-Einstein relation with the stick hydrodynamic boundary condition for the friction predicts that the translational diffusion of rodlike molecules in the direction parallel $\left(D_{\|}\right)$to the long axis is only twice as fast as that in the perpendicular $\left(D_{\perp}\right)$ direction. ${ }^{2,4,5}$

The prediction on the motions of a prolate ellipsoid is nearly identical when the aspect ratio $(\kappa)$ is greater than $2(\kappa$ is defined as the ratio of the lengths of major to minor axes). The explicit expressions for $D_{\|}$and $D_{\perp}$ for a rod-like polymer in a liquid of viscosity $\eta$ are given by ${ }^{2}$

$$
D_{\|}=\frac{k_{B} T \ln (L / b)}{2 \pi \eta L},
$$

$$
D_{\perp}=\frac{k_{B} T \ln (L / b)}{4 \pi \eta L}
$$

where $k_{B} T$ is the Boltzmann constant times the temperature, $L$ and $b$ are the length and the diameter of the rod, respectively, and for a rod, $\kappa=L / b$. Note that $D_{\|}$and $D_{\perp}$ are defined in terms of mean-square displacements along the $Z$ and $X$ - (or $Y$-) axes, respectively. The $Z$ axis is chosen parallel to the long (major) axis of the rod (prolate ellipsoid) at time $t=0$. According to the above equations. $D_{\|}$and $D_{\perp}$ vary identically with $L$ and $\kappa$. Thus, the ratio $D_{\|} / D_{\perp}$ is independent not only of the length $L$, but also of the aspect ratio $\kappa$ of the rod. Another prediction is that if we fix $L$ and vary $b$, then both $D_{\|}$and $D_{\perp}$ increase as $\ln \kappa$. These are remarkable predictions (expected to be valid for any $\kappa>1$ ) which should be tested against experiments and simulations, although we are not aware of any such studies.

On the other hand, Evans and co-workers, in a very important and elegant contribution, have shown that for diffusion of long ellipsoids in a liquid, the use of the stick hydrodynamic boundary condition can be completely misleading. ${ }^{4}$ These authors showed by numerical solution of the NavierStokes hydrodynamic equations with the slip boundary condition that the motion along the parallel direction can become completely decoupled from that along the perpendicular direction and that the ratio between the two diffusion coefficients should approach $\kappa$ for large $\kappa^{4,5}$

Such a huge difference in the predictions between the stick and slip boundary condition is of course not new. A similar difference is found for the rotational friction on a sphere. ${ }^{6}$ In the latter case the slip boundary condition gives a

${ }^{a)}$ Electronic mail: bbagchi@sscu.iisc.ernet.in 
value of zero for the friction, while the stick boundary condition gives a value equal to $8 \pi \eta R^{3}$ where $\eta$ and $R$ are the viscosity of the liquid and the radius of the sphere, respectively. Accurate knowledge about the relative magnitudes of $D_{\|}$and $D_{\perp}$ is required for several problems of great current interest. For example, a preferential motion in the direction along the major axis is essential for reptation of rod-like polymers. ${ }^{7}$ Similarly, the transport properties of polyelectrolyte solutions can be affected by the presence of needlelike motion of rod-like polyelectrolytes. ${ }^{3,8} \mathrm{~A}$ recent theoretical study of the excess viscosity of rod-like polyelectrolytes invoked the anisotropic diffusion of the rods to explain the experimentally observed maximum at low polyelectrolyte concentration. ${ }^{8}$ It is thus very important to find whether the slip or the stick boundary condition provides the more appropriate description of the solvent friction on rod-like molecules.

One more aspect of interest here is the length $(L)$ dependence of the rotational correlation time $\tau_{2 R}$ of long molecules, which, according to stick hydrodynamic theory, should vary as $L^{3} / \ln \kappa$. Thus, within this theory, we have the following relation between the total diffusion coefficient $D_{T}$ and the rotational correlation time $\tau_{2 R}:{ }^{7}$

$$
D_{T} \tau_{2 R} \approx L^{2} \text {. }
$$

We have carried out detailed molecular dynamics simulations to test the above predictions. This study can be considered a continuation of our earlier study, ${ }^{9}$ where the parallel and the perpendicular components of the diffusion coefficient were obtained from the respective velocity time correlation functions. Thus, the previous study could not study the crossover from the anisotropic to the isotropic diffusion. In addition, the previous study was restricted to smaller values of the aspect ratio, which was varied by changing only the length of the ellipsoid. Also, no detailed test with hydrodynamics was carried out.

The results of the present study are rather interesting. First, we find that at short to intermediate times, the motion of ellipsoids is anisotropic and primarily needlelike - the molecules prefer to move parallel to their long axis. The ratio of these two diffusion constants $\left(D_{\|}\right.$and $\left.D_{\perp}\right)$ approaches $\kappa$, suggesting a decoupling of $D_{\|}$from the length of the ellipsoid. This is a clear indication of the validity of the slip boundary condition. As expected, the diffusion becomes isotropic in the long time, where the total diffusion coefficient is given by $D_{\|}+2 D_{\perp}$. However, the crossover from the anisotropic to the isotropic diffusion is surprisingly sharp and clear in most cases. The time at which this crossover takes place is found to correlate with the orientational correlation time.

The organization of the rest of the paper is as follows. In the next section we discuss the model and present the simulation details. In Sec. III, we present the results and discuss their significance. Section IV concludes with a summary of the results.

\section{THE MODEL AND THE SIMULATION DETAILS}

In this paper, we present molecular dynamics (MD) simulations of $D_{\|}$and $D_{\perp}$ and also rotational correlation time $\tau_{2 R}$ of tagged, isolated, thin Gay-Berne ellipsoids ${ }^{10}$ in a liquid of Lennard-Jones spheres. We have termed this model "'ellipsoids in the sea of spheres,' or the ESS model. The interaction between the particles is modeled by different potentials. The interaction between the spheres is given by the Lennard-Jones potential

$$
U_{\mathrm{LJ}}=4 \epsilon_{0}\left[\left[\frac{\sigma_{0}}{r}\right]^{12}-\left[\frac{\sigma_{0}}{r}\right]^{6}\right],
$$

where $\sigma_{0}$ is the molecular diameter and $r$ is the interparticle distance; $\epsilon_{0}$ is the energy parameter. The interaction energy between two ellipsoids with arbitrary orientations is assumed to be given by the Gay-Berne potential ${ }^{10}$

$$
\begin{aligned}
U_{\mathrm{GB}}= & 4 \epsilon\left(\hat{r}, \hat{u}_{1}, \hat{u}_{2}\right)\left[\left[\frac{\sigma_{0}}{r-\sigma\left(\hat{r}, \hat{u}_{1}, \hat{u}_{2}\right)+\sigma_{0}}\right]^{12}\right. \\
& \left.-\left[\frac{\sigma_{0}}{r-\sigma\left(\hat{r}, \hat{u}_{1} \hat{u}_{2}\right)+\sigma_{0}}\right]^{6}\right],
\end{aligned}
$$

where $\hat{u}_{1}$ is the axial vector of the molecule $i$ and $\hat{r}$ is the vector along the intermolecular vector $r=r_{2}-r_{1}$, where $r_{1}$ and $r_{2}$ denote the centers of mass of molecule 1 and 2, respectively. $\sigma\left(\hat{r}, \hat{u}_{1}, \hat{u}_{2}\right)$ and $\epsilon\left(\hat{r}, \hat{u}_{1}, \hat{u}_{2}\right)$ are the orientationdependent range and strength parameters, respectively. $\sigma$ and $\epsilon$ depend on the aspect ratio $\kappa$. Finally, the interaction between a sphere and an ellipsoid is accounted for by a modified GB-LJ potential given below ${ }^{9,11}$

$$
U_{\mathrm{GBLJ}}=4 \epsilon_{0}\left[\left[\frac{\sigma(\theta)}{r}\right]^{12}-\left[\frac{\sigma(\theta)}{r}\right]^{6}\right],
$$

with

$$
\sigma(\theta)=\frac{(b / 2+R)}{\sqrt{1+X^{2} \cos ^{2} \theta}},
$$

where $\theta$ is the angle between the major axis of the ellipsoid and the vector joining the centers of the sphere and the ellipsoid.

$$
\begin{aligned}
& R=\sigma_{0} / 2, \\
& X=\sqrt{\frac{\bar{a}^{2}-b^{2} / 4}{\bar{a}^{2}+R^{2}}},
\end{aligned}
$$

and

$$
\bar{a}=\sqrt{\left(b^{2} / 4+R^{2}\right)\left(\frac{L / 2+R}{b / 2+R}\right)^{2}-R^{2}} .
$$

Molecular dynamics simulations, with constant number $(\mathrm{N})$, volume (V) and energy (E) constraints, were carried out in a cubicbox by imposing the periodic boundary conditions, for a system containing 492 particles out of which $10 \mathrm{~GB}$ ellipsoids were placed far from each other in the sea of the remaining $482 \mathrm{LJ}$ spheres, except for aspect ratios 3.5 and 4 where we considered $950 \mathrm{LJ}$ spheres. All the quantities in the simulation were scaled to appropriate units; that is, the density by $\sigma_{0}^{-3}$, the temperature by $\epsilon / k_{B}$, and the time by $\checkmark\left(m \sigma_{0}^{2} / \epsilon\right)$ - the scaled quantities are denoted by $\rho^{*}, T^{*}$, and $t^{*}$, respectively. We have simulated the following thermodynamic state $\left(\rho^{*}, T^{*}\right)$ points: $(0.7,1.0) ;(0.5,1.0) ;(0.4,1.0)$; $(0.75,1.25)$. Most of the results presented here are for 


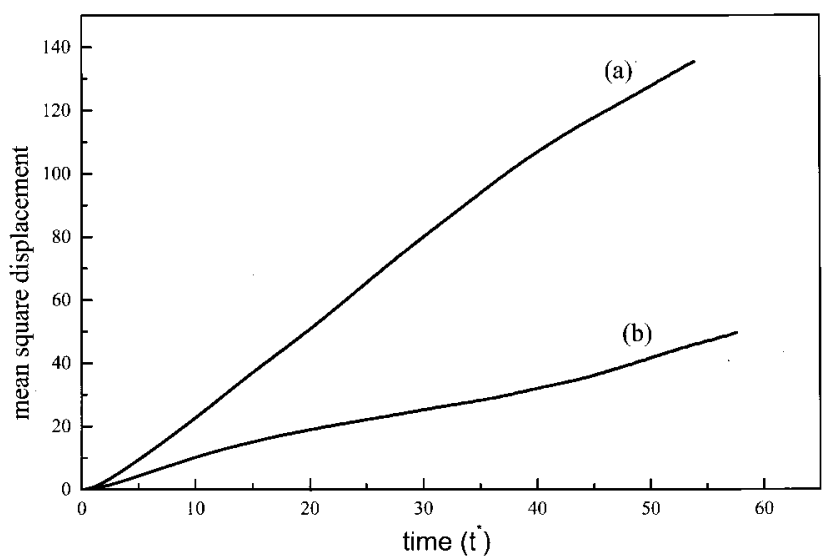

FIG. 1. The mean-square displacement of the (a) parallel and (b) perpendicular components of an ellipsoid with aspect ratio $[(\kappa)]$ equal to 6 . The length of the major axis is $3 \sigma_{0}$ and that of the minor axis is $0.5 \sigma_{0}$. The reduced solvent density, $\rho^{*}=0.4$, and the reduced temperature, $T^{*}=1.0$.

$\rho^{*}=0.7$ and $T^{*}=1.0$. The time step $\Delta t$ used was 0.002 . The system was equilibrated for $1 \times 10^{5}$ time steps and the averages were obtained over $2 \times 10^{5}$ time steps. In each case we ran at least three independent simulations and took the average. Ellipsoids with aspect ratio between 1.5 and 10 were generated by two methods. First, we increased the radius of the major axis, $L$, while keeping that of the minor axis, $b$, fixed. By this method we could go only up to $\kappa=4$, due to the obvious size constraints. The other method we employed was to fix $L / \lambda_{0}$ and then make $b / \sigma_{0}$ smaller. We generated $\kappa$ values equal to $2,4,6$ (any in a preliminary study even 10) by this method. By the time the aspect ratio is 10 , we have indeed very thin ellipsoids. It is interesting to find that such thin and long ellipsoids also have well-defined diffusion constants. Note that while the former method mimics real polymers, the second one is also of interest, particularly in checking theoretical predictions. The simulation results are discussed below.

\section{RESULTS AND DISCUSSION}

In Fig. 1 we plot the parallel and the perpendicular (with respect to the prolate's orientation at time $t=0$ ) mean-square displacements of an ellipsoid of aspect ratio 6, at somewhat lower density $\left(\rho^{*}=0.4\right)$ - higher densities show similar behavior. Three distinct regions in the respective mean-square displacement are seen for both the components. At very short times, the motion is ballistic. This is followed by a substantial duration when the diffusion is anisotropic. During this time, the motion of the prolate can indeed be termed needlelike, because the motion along the parallel direction is much faster than that along the perpendicular direction. In the long time the diffusion becomes isotropic (that is, both the parallel and the perpendicular motions have the same diffusion constant) and the total isotropic diffusion coefficient $D$ is given by $D_{\|}+2 D_{\perp}$.

In Fig. 2, we show the comparison of the simulation results with the prediction of the stick boundary condition. In this figure, we changed the aspect ratio $\kappa$ by varying the diameter $b$ of the minor axis. Note the weak dependence of

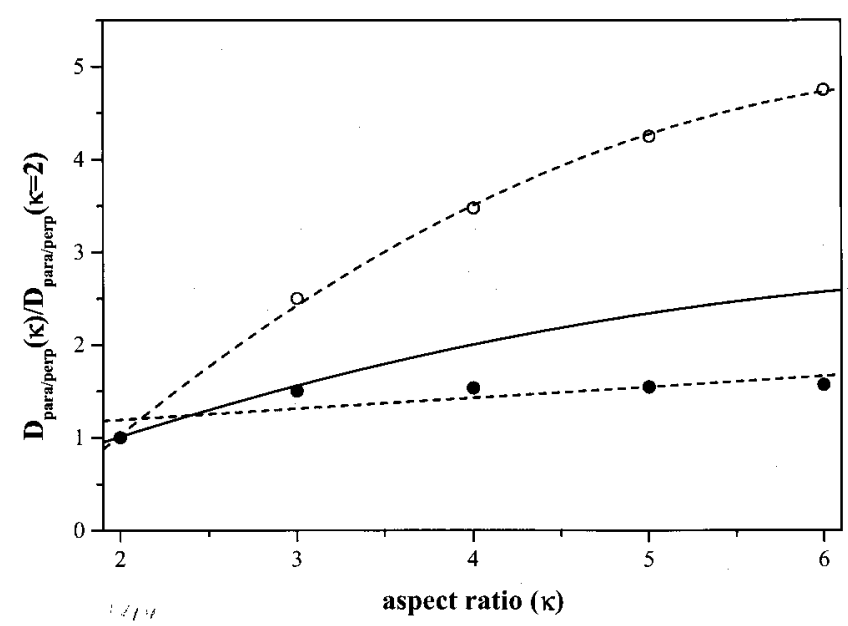

FIG. 2. The comparison of the ratio, $\left[D_{\|}(\kappa)\right] /\left[D_{\|}(\kappa=2)\right]$ and $\left[D_{\perp}(\kappa)\right] /\left[D_{\perp}(\kappa=2)\right]$ with the prediction of the stick boundary condition (Doi-Edwards) is plotted against $\kappa$. The open circles represent the parallel component and the filled ones are for the perpendicular. The solid line is the prediction of the stick hydrodynamic boundary condition. $\rho^{*}=0.7$ and $T^{*}=1.0$.

$D_{\perp}$ on $\kappa$, in defiance of Doi-Edwards theory which also fails to explain $\kappa$ dependence of $D_{\|}$. Similar results were obtained earlier by Evans et $a l^{4,5}$ for hard ellipsoids, but in a liquid of ellipsoidal molecules.

In Fig. 3, we plot the ratio, $D_{\|} / D_{\perp}$ against the aspect ratio $\kappa$-the latter is now varied by changing both $L$ and $b$. One clearly sees the decoupling of the motion parallel to the major axis from the length of the ellipsoid, signalling the breakdown of the stick hydrodynamic boundary condition. It is truly interesting to find how closely the results follow the prediction of the slip boundary condition!

We found that for all the systems studied the decay of the orientational correlation function $\left\langle P_{l}(\cos (\theta(0))) P_{l}(\cos (\theta(t)))\right\rangle$ (where $P_{l}$ is the Legendre polynomial of order $l$ and $\theta(t)$ is the angle that the major axis

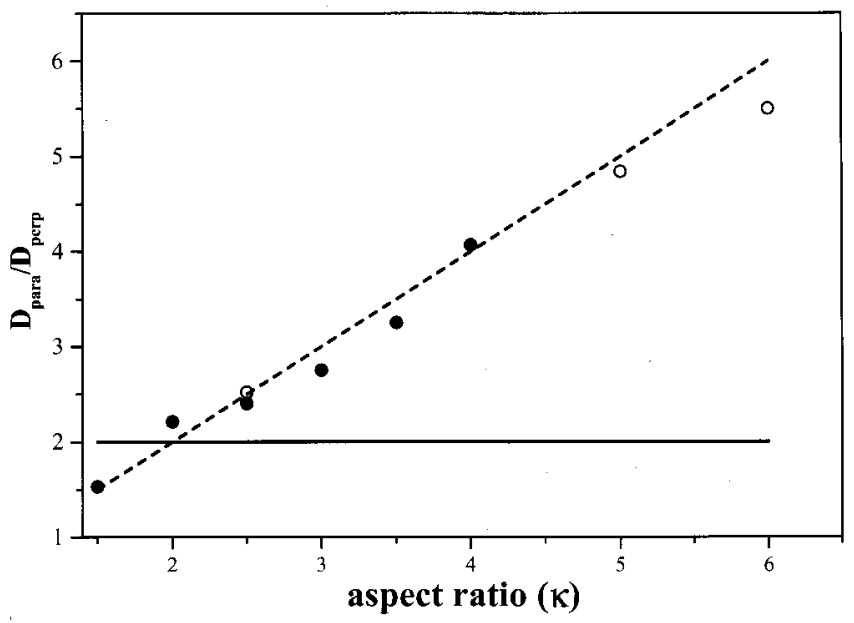

FIG. 3. The simulated ratio, $D_{\|} / D_{\perp}$, is plotted against the aspect ratio, $\kappa$. The prediction of hydrodynamics with stick boundary condition ([2]) is shown by the solid line and that of the slip boundary condition ([4)] by the dashed line. $\rho^{*}=0.7$ and $T^{*}=1.0$. 


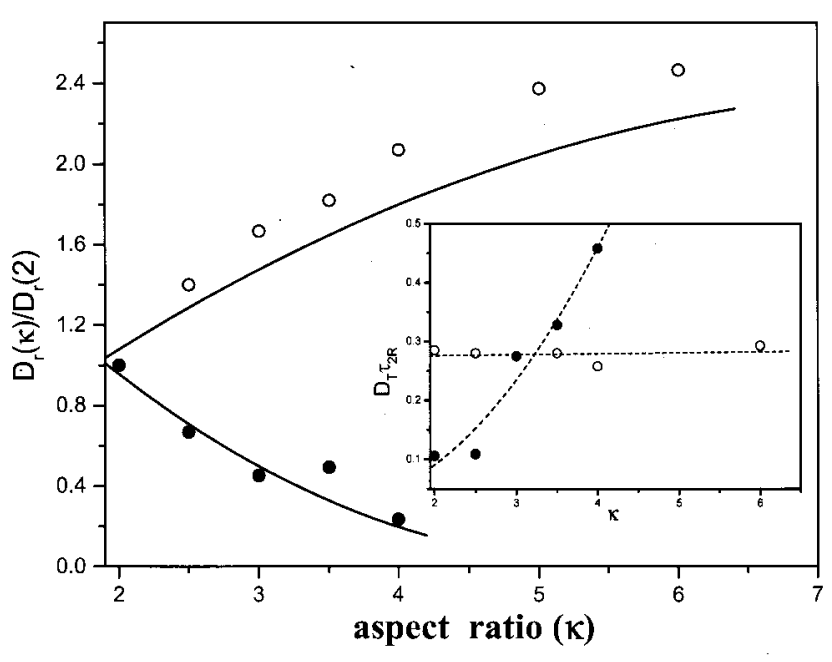

FIG. 4. The main figure depicts the aspect ratio dependence of the simulated rotational diffusion coefficient, $D_{R}$. The simulated values of the ratio $\left[D_{R}(\kappa)\right] /\left[D_{R}(\kappa=2)\right]$ have been compared with the prediction of DoiEdwards theory (solid lines). The inset shows the product of the total diffusion coefficient, $D_{T}$, with the rotational relaxation time. The upper dashed line is the quadratic fit, while the lower dashed line is a linear fit. Both in the main figure and in the inset, the open circles represent simulated results obtained by varying $b$ while the filled circles represent those obtained by varying the length $L$ of the major axis. $\rho^{*}=0.7$ and $T^{*}=1.0$.

makes with the $Z$ axis at time $t$ ) is exponential-like. The decay time constants are found to follow the Debye $l(l+1)$ behavior.

In Fig. 4, we show the variation of the ratio $\left[D_{R}(\kappa)\right] /\left[D_{R}(\kappa=2)\right]$ with the aspect ratio $\kappa$, and also the hydrodynamic prediction, with the stick boundary condition. Note that here $D_{R}=1 / 6 \tau_{2 R}$. As can be seen from Fig. 4, the agreement with the hydrodynamic theory is quite satisfactory. In the inset, we show the $\kappa$ dependence of the product of the total diffusion coefficient, $D_{T}=D_{\|}+2 D_{\perp}$ and the rotational relaxation time $\tau_{2 R}$. The values have been obtained by varying either the length $L$ (filled circles) or the diameter $b$ (the open circles). The product $D_{T} \tau_{2 R}$ is plotted against the aspect ratio $\kappa$ for both long and thin ellipsoids. It is observed that for thin ellipsoids, the product is independent of the aspect ratio and is found to scale as $L^{2}$, as envisaged in Eq. (3). This is somewhat surprising and is under further investigation.

What is the physical reason for the slip-like hydrodynamic boundary condition for translational motion along the major axis of the ellipsoid? It seems plausible that the motion along the long axis involves much less displacement of the solvent molecules and the ellipsoid can slip past the solvent molecules, thus setting up a hydrodynamic velocity field well described by the slip boundary condition. The motion along the perpendicular direction of course remains coupled to the size of the polymer. It is a common wisdom that for large molecules, the stick boundary condition is more appropriate than the slip. The resolution of the paradox probably lies in the earlier study of Zwanzig, ${ }^{12}$ who showed that for a large rough cylinder (radius $\sim 500 \AA$ ) the stick and the slip boundary conditions become identical. This is because the solvent molecules are "caught up" in the indentations of the rough surface and are carried along. Real systems may interpolate between the two extremes described by the slip and the stick boundary conditions. Theoretically one might be able to approach the problem by using the anisotropic bead model studied by Pastor and Zwanzig, ${ }^{13}$ who pointed out that the failure of Doi-Edwards theory arises from their use of the Burgers-Kirkwood-Riseman theory, ${ }^{14,15}$ which uses a scalar friction coefficient. Pastor and Zwanzig developed a scheme which allows the use of a tensorial friction coefficient in the numerical solution of hydrodynamic equation for the total friction of the bead chain. It should be possible to model the slip boundary condition by assuming that the tangential component of the bead friction tensor is zero. It will be worthwhile to carry out a simulation check of this procedure. Thus, further computer simulations and microscopic studies, and of course experiments, are required to understand this aspect of the problem.

\section{CONCLUSION}

To summarize, it is shown, we believe for the first time, that the motion of long ellipsoids in liquids is needlelike at short to intermediate times. The ratio $D_{\|} / D_{\perp}$ is found to increase as $\kappa$, while the Doi-Edwards theory predicts this ratio to be a constant, independent both of length $L$ of the ellipse and of the aspect ratio, $\kappa$. The motion in the perpendicular direction is weakly dependent on the aspect ratio, again in disagreement with Doi-Edwards theory. The time at which the crossover from the needlelike to the isotropic diffusion is found to correlate with the orientational relaxation times of the prolates.

\section{ACKNOWLEDGMENTS}

We would like to thank Professor Robert Zwanzig for several valuable comments. We thank Mr. G. Srinivas and Dr. S. Bhattacharyya for help and discussions. This work was supported in part by the Department of Science and Technology, and the Council of Scientific and Industrial Research, India.

${ }^{1}$ B. J. Berne and R. Pecora, Dynamic Light Scattering (Wiley, New York, 1976), Chap. 7.

${ }^{2}$ M. Doi and S. F. Edwards, The Theory of Polymer Dynamics (Clarendon, Oxford, 1986), Chap. 8.

${ }^{3}$ S. Forster and M. Schmidt, Adv. Polym. Sci. 120, 50 (1995).

${ }^{4}$ S. Tang and G. T. Evans, Mol. Phys. 80, 1443 (1993).

${ }^{5}$ S. Tang, G. T. Evans, C. P. Mason, and M. P. Allen, J. Chem. Phys. 102, 3794 (1995)

${ }^{6}$ C. M. Hu and R. Zwanzig, J. Chem. Phys. 60, 4354 (1974).

${ }^{7}$ M. Fixman, Phys. Rev. Lett. 54, 337 (1985).

${ }^{8}$ B. Bagchi and A. Yethiraj (unpublished).

${ }^{9}$ S. Ravichandran and B. Bagchi, J. Chem. Phys. 111, 7505 (1999).

${ }^{10}$ J. G. Gay and B. J. Berne, J. Chem. Phys. 74, 3316 (1981).

${ }^{11}$ The potential has been developed and tested in collaboration with Dr. A. Perera of LPTL, Univ. P. et M. Curie, Jussieu, Paris 75252 (private communication). This potential was used in Ref. 9 and shown to give consistent results.

${ }^{12}$ R. Zwanzig, J. Chem. Phys. 68, 4325 (1978).

${ }^{13}$ R. W. Pastor and R. Zwanzig, J. Chem. Phys. 90, 5729 (1989).

${ }^{14}$ S. J. M. Burgers, Second Report on Viscosity and Plasticity (Amsterdam Academy of Science, Nordemann, Amsterdam, 1938).

${ }^{15}$ J. G. Kirkwood and J. Riseman, J. Chem. Phys. 16, 565 (1948). 